\title{
Curvas de crescimento em bovinos da raça Indubrasil criados no Estado do Sergipe ${ }^{1}$
}

\author{
Growth rate curves of Indubrasil cattle raised at Sergipe State
}

\author{
Laaina de Andrade Souza ${ }^{2 *}$, Dirlane Novais Caires ${ }^{2}$, Paulo Luiz Souza Carneiro ${ }^{3}$, Carlos Henrique Mendes \\ Malhado $^{3}$ e Raimundo Martins Filho ${ }^{4}$
}

\begin{abstract}
Resumo - O objetivo deste estudo foi analisar modelos não-lineares para descrever o crescimento em animais da raça Indubrasil no Estado do Sergipe. Após a definição do modelo de melhor ajuste, calculou-se a taxa de crescimento absoluto e avaliou-se a influência de fatores ambientais (sexo, fazenda, mês e ano de nascimento) sobre os parâmetros da curva. Utilizaram-se dados de, no mínimo, 8 pesagens, obtidas a intervalos de aproximadamente 90 dias, do nascimento aos 2 anos de idade de 1.201 bovinos, provenientes da Associação Brasileira dos Criadores de Zebu (ABCZ). Os modelos não-lineares utilizados foram: Brody, Von Bertalanffy, Richards, Logístico e Gompertz. O modelo Logístico apresentou melhor ajuste seguido dos modelos Gompertz e Von Bertalanffy. O peso assintótico $(A)$ e a taxa de maturidade $(k)$ estimada pelo modelo Logístico foram 427,87 kg e 0,0052, respectivamente. Constatou-se acentuado decréscimo da taxa de crescimento absoluto na fase pós-desmama, devendo ser adotado um regime alimentar adequado para acompanhar as exigências de nutrientes dos animais nessa fase. Os efeitos de ambiente influenciaram significativamente $(\mathrm{P}<0,05)$ os parâmetros $A$ e $k$. A correlação estimada entre os parâmetros $A$ e $k$ foi negativa $(-0,62)$, indicando que os animais mais precoces possuem menor probabilidade de atingir pesos elevados à idade adulta.
\end{abstract}

Palavras-chave - Bovino-crescimento. Bovino de corte-melhoramento genético.

\begin{abstract}
The objective of this study was to analyze nonlinear models to describe growth of Indubrazil cattle raised in Sergipe State. After defining the best adjusted model, it was evaluated the influence of environmental effects (sex, farm, month and year of birth) over mature weight $(A)$ and maturation rate $(k)$. Data from 1,201 animals weighed in nearly 90-day intervals from birth up to two years of age were used, based on records from the Brazilian Association of Zebu Breeders (ABCZ). The models utilized were Brody, Von Bertalanffy, Richards, Logistic and Gompertz. The Logistic model presented best adjustment followed by Gompertz and Von Bertalanffy models. The parameters $A$ (weight at maturity) and $k$ (maturation rate), obtained by the Logistic model were 427.87 and 0.0052 , respectively. An accentuated decrease in the absolute growth rate was observed after weaning and nutritional strategies should be performed to avoid the low weight gain at this stage. Environmental factors affected significantly the parameters $A$ and $k$. The correlation estimated between the parameter $A$ and $k$ was negative $(-0.62)$, indicating that fast-growing young animals are less likely to reach higher weights when adults.
\end{abstract}

Key words - Bovine-growth. Beef cattle-breeding.

\footnotetext{
* Autor para correspondência

${ }^{1}$ Recebido para publicação em 24/09/2009; aprovado em 17/09/2010

Pesquisa financiada pela Universidade Estadual do Sudoeste da Bahia-UESB

2Programa de Pós-Graduação em Zootecnia, PPZ/UESB, Itapetinga-BA, Brasil, laainaandrade@gmail.com, dinhanovais@yahoo.com.br ${ }^{3}$ Departamento de Ciências Biológicas, Universidade Estadual do Sudoeste da Bahia, Campus de Jequié, BA, Brasil, plscarneiro@gmail.com, carlosmalhado@gmail.com

${ }^{4}$ Universidade Federal do Ceará, Campus do Cariri, CE, Brasil, rmartinsfilho@yahoo.com.br
} 


\section{Introdução}

Em sistemas de produção de carne, os criadores estão cada vez mais conscientes da importância da avaliação do crescimento dos animais para melhor analisar e gerenciar a rentabilidade dessa atividade, sendo o crescimento uma função primordial, pois apresenta relação direta com a quantidade e a qualidade da carne, produto final da exploração (SILVA et al., 2004). Estudos relacionados ao crescimento dos animais de corte são importantes em programas de melhoramento genético, por permitir subsídios à seleção para precocidade e ganho de peso (LÔBO et al., 2006).

No Brasil, existem poucos estudos com pesos de todo o ciclo de vida dos zebuínos, desde o nascimento até a maturidade e, portanto, não existe uma descrição global do crescimento e não foi estabelecido o que possa ser considerado como padrão médio de crescimento para zebuínos (OLIVEIRA et al., 2000).

De acordo com Malhado et al. (2008), curvas de crescimento podem ser utilizadas para descrever o crescimento do animal ao longo do tempo, auxiliando no estabelecimento de programas alimentares e na definição da idade ótima de abate. As curvas de crescimento que relacionam o peso do animal com a sua idade utilizam modelos não lineares para condensar grande volume de informação em um pequeno conjunto de parâmetros que podem ser interpretados biologicamente (MCMANUS et al., 2003).

Os modelos mais utilizados para descrever o crescimento dos animais são modelos biológicos, como Brody (BRODY, 1945), Von Bertalanffy (BERTALANFFY, 1957), Richards (RICHARDS, 1959), Logístico (NELDER, 1961) e Gompertz (LAIRD, 1965). Segundo Santoro et al. (2005), os benefícios do uso desses modelos no melhoramento genético animal seriam a estimação dos parâmetros para as curvas e a identificação dos animais mais apropriados a determinados fins, como maior ganho de peso em uma fase específica da vida.

Identificado o modelo que melhor se ajuste ao padrão de crescimento, os parâmetros que o descrevem devem ser estudados, determinando os fatores que podem influenciá-los, como raça, sexo, tipo de nascimento, época e ano de nascimento e idade da mãe ao parto, possibilitando ajustes no padrão de crescimento (SARMENTO et al., 2006).

O objetivo deste trabalho foi analisar modelos não-lineares para descrever o crescimento em bovinos Indubrasil criados no Estado do Sergipe, e, após a definição do modelo de melhor ajuste, calcular a taxa de crescimento absoluto e avaliar a influência dos fatores de ambiente sobre os parâmetros estimados da curva.

\section{Material e métodos}

Para determinar a curva de crescimento, foram utilizados dados constantes do Controle de Desenvolvimento Ponderal da Associação Brasileira de Criadores de Zebu (ABCZ), referentes a 1.201 animais da raça Indubrasil criados a pasto, nascidos entre 1975 e 2006 no Estado de Sergipe. Foram utilizados dados de pesagens até 721 dias de idade.

Foram ajustados os modelos Brody, Von Bertalanffy, Richards, Logístico e Gompertz (TAB. 1) para se estimar o crescimento do animal e os parâmetros da curva, em que $\mathrm{Y}$ é o peso corporal à idade $t ; A$, o peso assintótico, sendo esse parâmetro interpretado como peso à idade adulta; $B$, uma constante de integração, relacionada aos pesos iniciais do animal. O valor de $B$ é estabelecido pelos valores iniciais de $Y$ e $t$. O $k$ é interpretado como taxa de maturação, que é a mudança de peso em relação ao peso e à maturidade, ou seja, indicador da velocidade com que o animal se aproxima do seu tamanho adulto. O parâmetro $m$ é que dá forma à curva e, conseqüentemente, determina em que proporção do valor assintótico $(A)$ ocorre o ponto de inflexão da curva.

Tabela 1 - Forma geral dos modelos não-lineares

\begin{tabular}{cc}
\hline Modelo & Forma Geral \\
\hline Brody & $y=A\left(1-B e^{-k t}\right)+\varepsilon$ \\
Von Bertalanffy & $y=A\left(1-B e^{-k t}\right)^{3}+\varepsilon$ \\
Richards & $y=A\left(1-B e^{-k t}\right)^{m}+\varepsilon$ \\
Logístico & $y=A\left(1+B e^{-k t}\right)^{-m}+\varepsilon$ \\
Gompertz & $\mathrm{y}=\mathrm{Ae}^{-\mathrm{Be}-\mathrm{kt}}+\varepsilon$ \\
\hline
\end{tabular}

No caso dos modelos não-lineares, não é possível resolver o sistema de equações formado diretamente, como ocorre no caso linear, uma vez que a resolução do sistema é dependente dos próprios parâmetros a serem estimados. Dessa forma, usa-se um processo iterativo para obtenção das estimativas dos parâmetros, o qual começa com valores iniciais, atribuídos aos próprios parâmetros a serem estimados. Calcula-se, então, a soma de quadrado do erro e, a cada passo, obtém-se um conjunto de estimativas atualizadas até o procedimento convergir para um vetor final de estimativas, obtendo-se a soma mínima de quadrados do erro (SARMENTO et al., 2006). Os parâmetros dos modelos foram estimados pelo método de Gauss Newton, modificado por meio do procedimento NLIN do SAS (2003), usando o critério de convergência de $10^{-8}$.

Os critérios utilizados para seleção do modelo que melhor descreveu a curva de crescimento foram: quadrado 
médio do resíduo (QMR) - calculado dividindo-se a soma de quadrados do resíduo, pelo número de observações, que é o estimador de máxima verossimilhança da variância residual, para que se pudesse comparar o QMR dos diferentes modelos, visto que possuem números diferentes de parâmetros a serem estimados; coeficiente de determinação $\left(\mathrm{R}^{2}\right)$ - calculado como o quadrado da correlação entre os pesos observados e estimados, através de uma análise de regressão linear considerando o peso predito pelo modelo como variável dependente e o peso observado do animal como variável independente; percentual de convergência $(\mathrm{C} \%)$ - considerando a existência ou não de convergência; análise gráfica dos modelos com o peso médio observado; e desvio médio absoluto dos resíduos (DMA) - estatística proposta por Sarmento et al. (2006) para avaliar a qualidade de ajuste, calculado como na Equação 1:

$\mathrm{DMA}=\frac{\sum_{i=1}^{n}\left|Y_{i}-\hat{Y}_{i}\right|}{n}$

Sendo que o $Y_{i}$ é o valor observado, $\hat{Y}_{i}$ o valor estimado e $n$ o tamanho da amostra. Quanto menor o valor do DMA, melhor o ajuste.

Depois de selecionado o modelo, calculou-se a taxa de crescimento absoluta (TCA), obtida a partir da primeira derivada do modelo ajustado, em relação ao tempo $(\partial Y / \partial t)$. A TCA é, na realidade, o ganho de peso obtido por unidade de tempo e, como, nesse caso, o tempo está em dia, representa o ganho de peso médio diário, estimado ao longo da trajetória de crescimento, ou seja, a taxa média de crescimento dos animais dentro da população.

$\mathrm{Na}$ etapa seguinte, foi avaliada a influência da fazenda (20), sexo (macho e fêmea), mês de nascimento (janeiro a dezembro) e ano de nascimento (1975 a 2006) sobre os parâmetros da curva do modelo selecionado, através do procedimento GLM do software SAS (2003). As correlações de Pearson entre os parâmetros foram estimadas pelo procedimento CORR do software SAS (2003).

\section{Resultados e discussão}

As estimativas dos parâmetros considerandose todos os dados, para cada modelo, e os critérios utilizados para avaliar o modelo que melhor descreveu a curva média de crescimento de bovinos Indubrasil são apresentados na Tabela 2.

O parâmetro $A$ representa a estimativa do peso assintótico, que é interpretado como peso adulto. Esse peso não é o máximo que o animal atinge, e sim o peso médio à maturidade livre das variações sazonais (BROWN et al., 1976). Ao comparar as estimativas do parâmetro $A$, obtidas pelos cinco modelos, percebe-se que o maior valor encontrado foi o do modelo Brody $(499,96)$, seguido pelos modelos Von Bertalanffy $(485,33)$, Richards $(468,09)$, Gompertz $(440,12)$ e Logístico $(427,87)$. Santoro et al. (2005), estudando zebuínos da raça Guzerá criados no Estado de Pernambuco encontraram valores inferiores de $A$ para os modelos Brody $(441,11)$, Von Bertalanffy $(419,50)$, Gompertz $(405,41)$ e Logístico $(401,35)$, e superiores para o modelo Richards $(485,30)$.

O parâmetro $k$ representa a taxa de maturidade do animal e indica a velocidade de crescimento para atingir o peso assintótico. Na estimativa desse parâmetro, observou-se que o modelo Richards $(0,0826)$ foi superior aos modelos Logístico $(0,0052)$, Gompertz $(0,0044)$, Von Bertalanffy $(0,0033)$ e Brody $(0,0022)$. Santoro et al. (2005) estimaram resultado semelhante para $k$ no modelo Brody $(0,0022)$, que apresentou menor valor para $k$, e maior valor para o modelo Logístico $(0,0070)$. Quanto maior o valor de $k$, maior a velocidade de crescimento do animal, apresentando maturidade precoce em relação aos animais com valor mais baixo de $k$ e peso inicial semelhante.

Com base no coeficiente de determinação $\left(\mathrm{R}^{2}\right)$ apresentado na Tabela 2, verificou-se que os modelos

Tabela 2 - Estimativas dos parâmetros $(A, B, k$ e $m)$, quadrado médio do resíduo (QMR), coeficiente de determinação $\left(\mathrm{R}^{2}\right)$ e percentual de convergência (C\%), desvio médio absoluto (DMA) de acordo com os modelos estudados

\begin{tabular}{ccccccccc}
\hline \multirow{2}{*}{ Modelo } & \multicolumn{7}{c}{ Parâmetros } \\
\cline { 2 - 9 } & $A$ & $B$ & $k$ & $m$ & QMR & $\mathrm{R}^{2}$ & $\mathrm{C}(\%)$ & DMA \\
\hline Brody & 499,961 & 0,9550 & 0,0022 & - & 440,56 & 0,958 & 74,17 & 13,68 \\
Bertalanffy & 485,337 & 0,5454 & 0,0033 & - & 327,94 & 0,970 & 92,91 & 12,49 \\
Richards & 468,098 & 0,9520 & 0,0826 & 0,0300 & 4714,56 & 0,604 & 77,49 & 34,14 \\
Logístico & 427,872 & - & 0,0052 & 2,9355 & 300,55 & 0,973 & 96,36 & 11,47 \\
Gompertz & 440,123 & - & 0,0044 & - & 473,12 & 0,957 & 94,00 & 12,88 \\
\hline
\end{tabular}


Logístico e Von Bertalanffy tiveram melhores ajustes que os demais, com valores de $97 \%$. Contudo, o modelo Logístico apresentou menor quadrado médio do resíduo (QMR) e menor desvio médio absoluto (DMA). O mesmo modelo apresentou maior percentual de convergência (96\%) em relação aos outros modelos. Souza e Bianchini Sobrinho (1994) frisaram que, quanto maior o número de convergências individuais em relação ao total de animais, melhor seria o modelo ajustado.

De acordo com os critérios analisados (coeficiente de determinação, quadrado médio do resíduo, percentual de convergência e desvio médio absoluto), o modelo Logístico foi o que melhor ajustou os dados e descreveu a curva do crescimento dos animais do rebanho estudado, seguido por Gompertz e Von Bertalanffy, quando comparados com os demais (FIG 1 e 2).

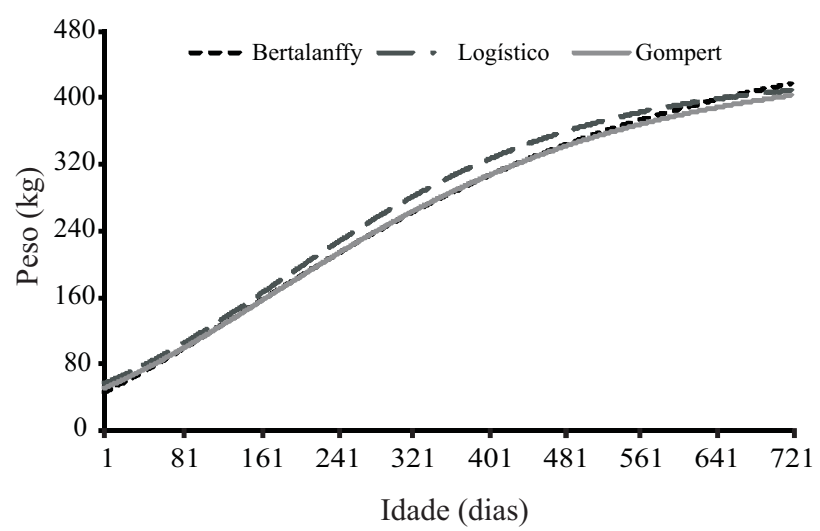

Figura 1 - Estimativa do peso em função da idade, ajustado pelos modelos Von Bertalanffy, Logístico e Gompertz em bovinos da raça Indubrasil criados no Estado do Sergipe

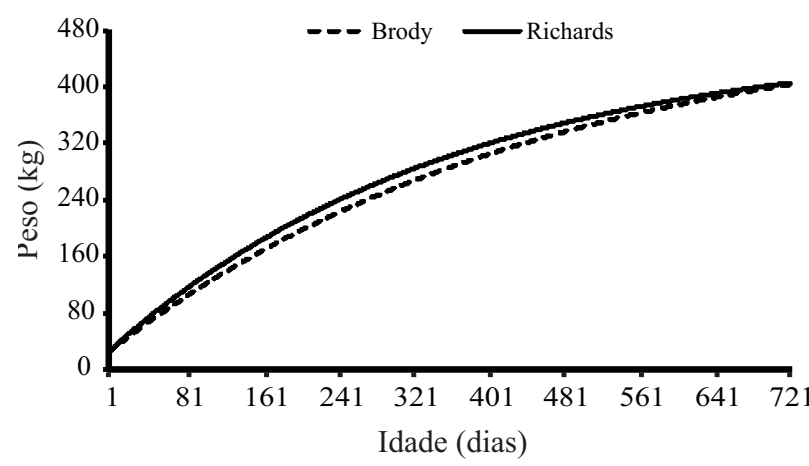

Figura 2 - Estimativa do peso em função da idade, ajustado pelos modelos Brody e Richardsem bovinos da raça Indubrasil criados no Estado do Sergipe
Vários estudos sobre curvas de crescimento têm sido realizados em bovinos e os resultados comprovam que apesar de vários modelos serem adequados, existem divergências entre eles. Segundo Silva et al. (2004), o modelo de Gompertz, sob o ponto de vista prático, é o mais indicado para descrever o crescimento de bovinos da raça Nelore, apresentando bom ajuste e maior porcentagem de convergência. Santoro et al. (2005) consideraram o modelo Logístico duplo o mais adequado para todas as raças (Guzerá, Nelore e Nelore Mocho) e sexos pela facilidade computacional, enquanto os modelos de Richards e Brody foram superiores quanto à interpretação biológica de seus parâmetros. Para Garnero et al. (2005) os modelos Von Bertalanffy, Brody, Gompertz e Logístico foram apropriados para estabelecer padrões médios de crescimento de fêmeas da raça Nelore, no entanto, considerando os aspectos de facilidade computacional e precisão de ajuste aos dados observados, os melhores resultados foram proporcionados pelo modelo Brody.

As taxas de crescimento absoluto (TCA), obtidas pela primeira derivada do modelo Logístico em função do tempo são apresentadas na Figura 3. As TCA foram crescentes até atingirem os máximos de 750 e $650 \mathrm{~g}$ por dia, para machos e fêmeas, respectivamente. Nessa fase, a taxa de crescimento é muito elevada e positiva, chegando ao máximo no ponto de inflexão da curva.

Os pontos de inflexão, ou seja, ponto que a função muda de crescente para decrescente, foram de 260 e 205 dias de idade, para machos e fêmeas respectivamente, com pesos de 223,72 kg (machos) e $174,28 \mathrm{~kg}$ (fêmeas). Percebe-se que os machos foram, aproximadamente, $28,5 \%$ mais pesados que as fêmeas. A partir do ponto de inflexão, a taxa de crescimento começa a diminuir em virtude de uma série de fatores que inibe progressivamente o crescimento, embora o animal não deixe de crescer (CRAPLET, 1966).

O decréscimo da taxa de crescimento após o período da desmama pode estar relacionado à mudança no tipo de alimentação, pois os animais deixam de consumir leite e passam a consumir exclusivamente pasto. Segundo Oliveira et al. (2006), quando os bezerros se aproximam do desmame, suas exigências nutricionais aumentam, se as exigências nutricionais dos bezerros são maiores que os nutrientes supridos pelo leite e/ou pasto, o crescimento será restringido.

As fêmeas tiveram maior velocidade de crescimento no período pré-desmame, época que ainda são dependentes do ambiente materno, principalmente da produção de leite da matriz. Contudo, seu ponto de inflexão é de aproximadamente dois meses antes dos machos, apresentando uma acentuada queda a partir desta idade. Sendo assim, como forma de amenizar o decréscimo 
da TCA após o desmame, deve ser adotado um regime alimentar adequado para acompanhar as exigências de nutrientes dos animais nessa fase. Essa informação é importante para auxiliar os produtores em programas alimentares e na definição da melhor idade ao abate.

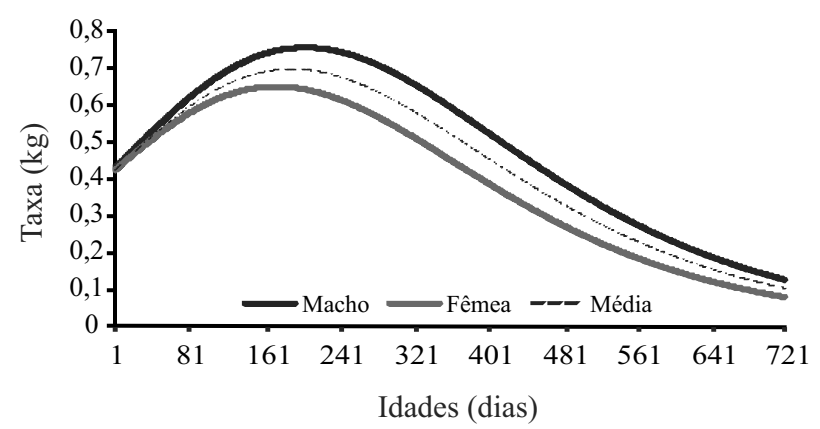

Figura 3 - Taxa de crescimento absoluto (TCA) estimado pela função Logística em bovinos da raça Indubrasil criados no Estado do Sergipe

Os efeitos de ambiente, de sexo, fazenda, mês e ano de nascimento influenciaram significativamente $(\mathrm{P}<0,05)$ os parâmetros $(A, k, m)$ da função Logística.

Os efeitos de ambiente influenciaram os parâmetros da curva Logística, corroborando com a noção de que o manejo e a correta nutrição influenciam diretamente o desenvolvimento do animal.

A influência do sexo sobre os parâmetros $A$ e $m$ foram maiores para os machos, com valores de $475,7 \mathrm{~kg}$ e 3,13, respectivamente. As fêmeas obtiveram valores de $392,1 \mathrm{~kg}$ para $A$ e 2,78 para $m$. O parâmetro $k$ foi ligeiramente superior para as fêmeas em relação aos machos, 0,0053 e 0,0050 respectivamente. Esse resultado indica que as fêmeas atingiram o peso assintótico mais rápido que os machos. Silva et al. (2004) encontraram resultados não significativos até o período do desmame, sendo que desse período aos 365 dias de idade constataram efeitos significativos, indicando que após o desmame os machos crescem com maior velocidade em relação às fêmeas.

Os efeitos fazenda, mês e ano de nascimento sobre os parâmetros evidenciam a influência das variações climáticas, tipo de manejo adotado, qualidade da pastagem e oferta de alimentos que agem diretamente sobre o desempenho dos animais. Segundo Martins et al. (2000) o efeito do rebanho e/ou da fazenda em que os animais são criados traduz diferenças relacionadas com o clima e manejo em geral, se tratando de rebanhos na mesma fazenda, este efeito pode refletir, em particular, em influências dos diferentes manejos alimentar e de programas de seleção postos em prática em cada rebanho.

A correlação entre as estimativas dos parâmetros $A$ e $k$ foi significativa $(\mathrm{P}<0,05)$ e igual a $-0,62$, corroborando aos achados de Santoro et al. (2005) e McManus et al. (2003).

Segundo Santoro et al. (2005) a correlação genética esperada entre o peso assintótico $(A)$ e a taxa de maturidade $(k)$ deve ser negativa, pois animais mais pesados tendem a ter taxa de maturação menor que animais mais leves, que, por sua vez, devem atingir a maturidade em idades menores. Contudo, nesse mesmo estudo, os autores relataram correlações positivas nas raças Guzerá (curva Richards) e Nelore Mocho (curvas Logística e Von Bertalanffy), sendo esse comportamento de difícil explicação, possivelmente em razão de problemas de amostragem.

\section{Conclusão}

1. O modelo Logístico apresentou melhor ajuste na descrição da curva de crescimento em bovinos da raça Indubrasil criados no Estado do Sergipe;

2. A taxa de crescimento absoluto teve uma acentuada queda após o período da desmama, portanto devem ser implementadas melhorias no manejo alimentar, visando melhorar o ganho de peso nessa fase;

3. A correlação estimada entre os parâmetros $A$ e $k$ foi negativa $(-0,62)$ indicando que a seleção para maior precocidade implicará a animais com menor peso adulto.

\section{Agradecimentos}

À FAPESB, CNPq e UESB pelo apoio financeiro.

\section{Referências}

BERTALANFFY, L. V. Quantitative laws in metabolism and growth. The Quarterly Reviem of Biology, v. 32, n. 03, p. 217230, 1957.

BRODY, S. Bioenergetics and growth. New York: Reinhhold Publication. 1945.1023 p.

BROW, J. E.; FITZHUGH JUNIOR, H. A.; CARTWRIGHT, T. C. A. A comparison of nonlinear models for describing weigtage relationships in cattle. Journal of Animal Science, v. 42, p. 810-818, 1976.

CRAPLET, C. Traité d'elevage modern. Tome III. La viande de bovins. Livre I. Paris: Vigot Frére Editeurs. 1966. 545 p. 
FREITAS, A. R. Curvas de Crescimento na Produção Animal. Revista Brasileira de Zootecnia, v. 34, n. 03, p. 786-795, 2005.

GARNERO, A. D. V. et al. Parâmetros genéticos da taxa de maturação e do peso assintótico de fêmeas da raça Nelore. Arquivo Brasileiro de Medicina Veterinária e Zootecnia, v. 57, n. 05, p. 652-662, 2005.

LAIRD, A. K. Dynamics of relative growth.Growth, v. 29, n. 03 , p. $249-263,1965$.

LÔBO, R. N. B. et al. Parâmetros genéticos de características estimadas da curva de crescimento de ovinos da raça Santa Inês. Revista Brasileira de Zootecnia, v. 35, n. 03, p. 1012 1019, 2006.

MALHADO, C. H. M. et al. Curva de crescimento em ovinos mestiços Santa Inês x Texel criados no Sudoeste do Estado da Bahia. Revista Brasileira de Saúde e Produção Animal, v. 09, n. 02, p. 210-218, 2008.

MARTINS, G. A. et al. Influência de fatores genéticos e de meio sobre o crescimento de bovinos da raça Nelore no Estado do Maranhão. Revista Brasileira de Zootecnia, v. 29, n. 01, p. 103-107, 2000.

McMANUS, C. et al. Curvas de crescimento de ovinos bergamácia criados no Distrito Federal. Revista Brasileira de Zootecnia, v. 32, n. 05, p. 1207-1212, 2003.

NELDER, J. A. The fitting of a generalization of the logistic curve. Biometrics, v. 17, p. 89-110, 1961.
OLIVEIRA, H. N. de; LÔBO, R. B.; PEREIRA, C. S. Comparação de modelos não-lineares para descrever o crescimento de fêmeas da raça Guzerá. Pesquisa Agropecuária Brasileira, v. 35, n. 09, p. 1843-1851, 2000.

OLIVEIRA, R. L. et al. Nutrição e manejo de bovinos de corte na fase de cria. Revista Brasileira de Saúde e Produção Animal, v. 07, n. 01, p. 57-86, 2006.

RICHARDS, F. J. A flexible growth function for empirical use. Journal of Experimental Botany, v. 10, n. 02, p. 290300, 1959.

SANTORO, K. R. et al. Estimativas de parâmetros de curvas de crescimento de bovinos zebu, criados no estado de Pernambuco. Revista Brasileira de Zootecnia, v. 34, n. 06, p. 2262-2279, 2005.

SARMENTO, J. L. R. et al. Estudo da curva de crescimento de ovinos Santa Inês. Revista Brasileira de Zootecnia, v. 35, n. 02, p. 435-442, 2006.

SAS INSTITUTE INC. Statistical Analysis System user's guide. Version 9.1 ed. Cary: SAS Institute, USA, 2003.

SILVA, N. A. M. et al. Curvas de crescimento e influência de fatores não-genéticos sobre as taxas de crescimento de bovinos da raça Nelore. Ciências Agrotécnicas de Lavras, v. 28, n. 03, p. 647-654, 2004.

SOUZA, J. C.; BIANCHINI SOBRINHO, E. Estimativas do peso de bovinos de corte, aos 24 meses, da raça Nelore usando curvas de crescimento. Revista Brasileira de Zootecnia, v. 23, n. 01, p. $85-91,1994$. 